\title{
Modelagem de um Data Mart para Leituras do Fluxo de Múons Captadas pelos Telescópios New-Tupi*
}

\author{
Lucas Bertelli ${ }^{1}$, Marcel N. de Oliveira ${ }^{2}$, Nívia Ferreira ${ }^{2}$, \\ Carlos E. Navia ${ }^{2}$, Daniel de Oliveira ${ }^{1}$ \\ ${ }^{1}$ Instituto de Computação - Universidade Federal Fluminense (IC/UFF) \\ ${ }^{2}$ Instituto de Física - Universidade Federal Fluminense (IF/UFF) \\ lucasbmeid.uff.br, \{paulista,nivia\}@fisica.if.uff.br \\ naviadif.uff.br, danielcmo@ic.uff.br
}

\begin{abstract}
The muon is the most abundant charged particle of cosmic radiation secondary at sea level. By reading the flow of muons, physicists are able to analyze and identify transient solar events that can impact our planet. New-Tupi telescopes are capable of reading the flow of muons that comes to Earth. These telescopes generate a large amount of data that needs to be queried by physicists. However, currently these telescopes store all the readings performed in binary files, which makes it difficult to elaborate queries on the data and its subsequent analysis. The goal of this paper is to propose a Data Mart for the New-Tupi telescope data, allowing for physicists to perform more complex queries in an easy way and with acceptable performance without having to rely on scripts or third-party programs to implement queries about the files.
\end{abstract}

Resumo. O múon é a partícula carregada mais abundante da radiação cósmica secundária ao nível do mar. Por meio da leitura do fluxo de múons, físicos são capazes de analisar e identificar eventos solares transientes, que podem gerar impactos em nosso planeta. Os telescópios New-Tupi são telescópios capazes de efetuar a leitura do fluxo de múons que chega à Terra. Esses telescópios geram um grande volume de dados que precisa ser consultado pelos físicos. Entretanto, atualmente tais telescópios armazenam todas as leituras realizadas em arquivos binários, o que dificulta a elaboração de consultas sobre os dados e sua posterior análise. O objetivo deste artigo é propor um Data Mart para os dados do telescópio New-Tupi, possibilitando aos físicos realizarem consultas mais complexas de forma fácil e com desempenho aceitável sem ter que recorrer à scripts ou programas de terceiros para implementar as consultas sobre os arquivos.

\section{Introdução}

Os raios cósmicos são partículas que atingem a atmosfera da Terra a todo instante. Eles podem ser de origem galáctica ou frutos da radiação solar. Os raios cósmicos podem ser divididos entre primários e secundários. Os primários possuem sua origem em fontes astrofísicas e os secundários são fruto da interação dos primários com o topo de nossa atmosfera, gerando assim um chuveiro de partículas. Nesses eventos, os píons [Lattes et al. 1947] decaem produzindo múons, neutrinos e raios gama através do seguintes processos: $\pi^{ \pm} \rightarrow \mu^{ \pm}+\bar{\nu}_{\mu}$ e $\pi^{0} \rightarrow \gamma+\gamma$.

O múon é a partícula carregada mais abundante da radiação cósmica secundária ao nível do mar e a única partícula com carga elétrica capaz de penetrar profundamente no subsolo

${ }^{*}$ Este artigo foi financiado parcialmente pelo CNPq, CAPES e FAPERJ 
terrestre [Zavattini 1975]. A medição do fluxo dessa partícula permite que cientistas estudem eventos solares transientes, tais como: erupções solares, ejeções de massa coronal (Coronal Mass Ejection - CME), radiações e tempestades geomagnéticas. Alguns desses eventos podem causar consequências para os modernos meios de comunicação e clima da Terra [Augusto et al. 2017]. Existem diversos detectores de partículas que são capazes de detectar raios cósmicos secundários, como os compõem a rede mundial de monitores de nêutrons (Network Neutron Monitor Database - NMDB ${ }^{1}$ ). A Universidade Federal Fluminense possui os telescópios de múons New-Tupi[Augusto et al. 2017]. O projeto New-tupi é um upgrade do projeto Tupi [Augusto et al. 2012a].

Os telescópios New-Tupi geram um grande volume de dados diário, aproximadamente 48.000 leituras do fluxo de múons (i.e., uma leitura a cada dois segundos aproximadamente). Atualmente, tais dados são armazenados em arquivos binários (*.DAT), que são gerados automaticamente pelos telescópios e gravados em um repositório na nuvem. De forma a extrair conhecimento útil de tais leituras (e.g., analisar a ocorrência de eventos solares transientes, por exemplo), os físicos necessitam realizar uma série de consultas complexas, muitas delas com agregações sobre tais dados (somatórios, médias, etc.) utilizando diferentes bins (intervalos de tempo como dias, semanas, meses), o que torna o trabalho tedioso e propenso a erros se realizado de forma manual ou por meio do uso de scripts.

Diante desse cenário, podemos observar diversos problemas e/ou limitações: Duplicidade de arquivos: dois aquivos podem ser referentes a mesma leitura diária; (ii) os físicos precisam implementar os cálculos necessários em uma ferramenta de análise, ainda que sejam análises simples e recorrentes; (iii) cada físico pode implementar cálculos diferentes, o que pode gerar inconsistência na análise, e (iv) as ferramentas de análise muitas vezes não oferecem um desempenho aceitável ou não são capazes de trabalhar com a quantidade de dados envolvida. Dessa forma, é fundamental que se possa fornecer capacidade analítica de um banco de dados para os físicos.

Nas últimas décadas, uma abordagem capaz de prover tal capacidade analítica, chamada de Data Warehouse (DW), tem sido amplamente utilizadas em diversos domínios [Golfarelli and Rizzi 2009], sejam eles acadêmicos ou comerciais [Inmon 1992]. DWs são bases de dados multidimensionais que integram informações de diversas fontes a fim de facilitar a análise de dados. Um DW reúne e consolida informações de diversos Data Marts (DM) [Inmon 1992], que são um subconjunto dos DWs que possuem um objetivo específico. Um DM é uma coleção de dados orientada por assuntos, variante no tempo, e não volátil. Uma das maiores vantagens dos DMs frente aos bancos de dados transacionais é que eles possuem dados previamente sumarizados, e.g., dados agregados por mês ou ano [Inmon 1992], o que acelera e facilita o processo de análise dos dados.

Dessa forma, o objetivo do presente artigo é desenvolver um DM, chamado TupiDM, para representar as leituras do fluxo de múons dos telescópios $\mathrm{New}$-Tupi. Assim, a ideia é fornecer uma maneira estruturada e simples de os físicos processarem e analisarem um grande conjunto de dados científicos e apoiar a sua tomada de decisão.

Esse artigo se encontra estruturado em 5 seções. A Seção 2 apresenta o referencial teórico. A Seção 3 apresenta a abordagem proposta chamada TupiDM. A Seção 4 apresenta avaliação experimental. A Seção 5 discute trabalhos relacionados, e, finalmente, a Seção 6 conclui o artigo e discute trabalhos futuros.

\footnotetext{
${ }^{1}$ http://www.nmdb.eu/
} 


\section{Referencial Teórico}

Essa seção apresenta alguns dos conceitos importantes para a compreensão desse artigo como modelagem dimensional e os telescópios New-tupi.

\subsection{Modelagem Dimensional}

Segundo [Kimball and Ross 2002], a modelagem dimensional é uma técnica de projeto de bancos de dados que visa apoiar consultas analíticas. Faz-se uso de redundâncias planejadas dos dados para melhorar o desempenho das consultas [Kimball and Ross 2002, Inmon 1992]. O modelo dimensional de um banco de dados é composto pelas tabelas Fato com suas respectivas Dimensões. As dimensões podem ser compartilhadas por tabelas fato diferentes. Existem dois modelos de implementação e um banco de dados dimensional: o Modelo Estrela [Kimball and Ross 2002] e o Modelo Floco de Neve [Inmon 1992]. O Modelo Estrela possui a tabela fato centralizada com as suas respectivas dimensões no seu entorno. Nesse modelo, a tabela fato possui chaves estrangeiras para todas as suas dimensões, sendo um modelo desnormalizado. O Modelo Floco de Neve é uma variação do Modelo Estrela, no qual todas as dimensões são normalizadas, fazendo com que sejam geradas quebras na tabela original ao longo de hierarquias existentes em seus atributos.

Um DW é constituído pela união dos DMs. Assim, como nos DMs, um DW preferencialmente deve ser modelado de forma dimensional, pois em comparação com um banco de dados transacional e normalizado, a modelagem dimensional produz modelos mais previsíveis e compreensíveis, facilitando a utilização e assimilação pelos usuários finais (no contexto desse artigo, os físicos), além de possibilitar consultas com alto desempenho [Kimball and Ross 2002]. Portanto, a modelagem dimensional possui uma estrutura simplificada, mais próxima da visão que o físico tem do seu domínio, facilitando assim a compreensão, de forma que os próprios físicos possam criar suas consultas. Apesar de terem uma estrutura diferente de bancos de dados transacionais, os bancos de dados dimensionais podem ser modelados sobre Sistemas de Gerência de Bancos de Dados (SGBDs) relacionais como o mySQL ou o PostsgreSQL.

\subsection{Os Telescópios New-Tupi}

Os telescópios de múons New-Tupi estão localizados no Instituto de Física da Universidade Federal Fluminense $\left(22.9^{\circ} \mathrm{S}, 43.2^{\circ} \mathrm{W} ; 5 \mathrm{~m}\right.$ acima do nível do mar). Os telescópios são constituídos por dois detectores fixos e outros dois que podem ser orientados de modo a detectar partículas provenientes de uma determinada direção [Augusto et al. 2017]. Os telescópios New-Tupi são construídos a partir de quatro detectores de partículas idênticos. Cada detector é construído com base em um cintilador plástico (Eljen EJ-208) de tamanho $(150 \mathrm{~cm} \times 75 \mathrm{~cm} \times 5 \mathrm{~cm})$ e uma fotomultiplicadora (Hamamatsu R877) de $127 \mathrm{~mm}$ de diâmetro, colocado dentro de uma caixa de formato piramidal truncada com quatro inclinações. O conjunto do detector é conectado à base fotomultiplicadora Ortec ScintiPack $^{T M}$ (Modelo 296).

Quando o múon atravessa o cintilador, este emite luz fluorescente que é captada pela fotomultiplicadora. A fotomultiplicadora converte a luz de baixa intensidade em um sinal elétrico, que é pré-amplificado até uma amplitude suficiente para facilitar uma posterior análise. A Figura 1 (esquerda) mostra uma fotografia dos telescópios $\mathrm{New}$-Tupi. Os quatro detectores são colocados em pares, com os detectores T1 e T2 no topo, e B1 e B2 no fundo, como mostrado na Figura 1 (direita). Este layout permite medir o fluxo de múons a partir de três direções, a vertical (zênite), oeste e leste (com uma inclinação de 45 graus). Os telescópios registram a taxa de coincidência para a incidência vertical usando os pares de 
detectores (T1, B1) e (T2, B2), bem como as coincidências cruzadas entre T1 e B2 (incidência oeste) e os T2 e B1 (incidência leste). A separação entre os detectores (vertical e horizontal) é $2,83 \mathrm{~m}$.

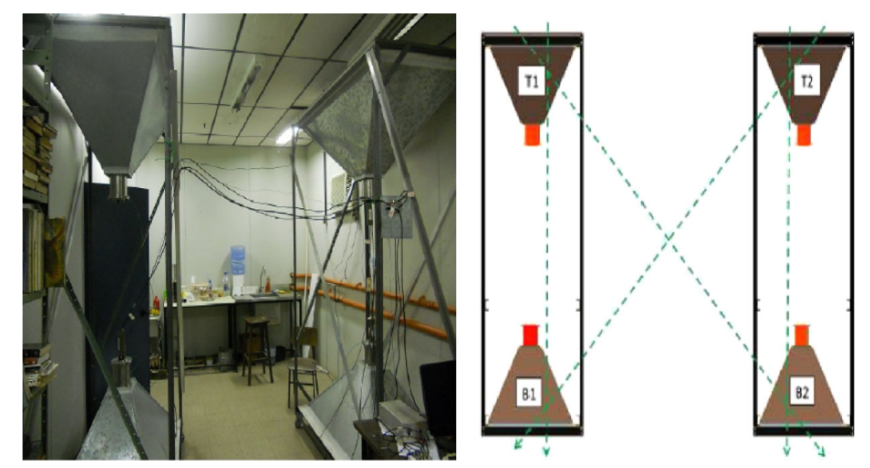

Figura 1. Esquerda: Fotografia dos telescópios New-Tupi. Direita: esquema geral do telescópio New-Tupi.

Os telescópios são automatizados e funcionam continuamente. Seus resultados ajudam a fomentar uma área emergente de estudos conhecida como clima espacial. Eles trabalham de forma sincronizada para medir continuamente o fluxo de partículas derivadas da radiação do Sol, investigando as possíveis relações entre os ciclos solares e as variações climáticas da Terra [Augusto et al. 2017]. Um exemplo do funcionamento do $\mathrm{New}$-Tupi pode ser visto na Figura 2. A Figura 2 mostra o efeito do nível do solo da segunda maior tempestade geomagnética do atual ciclo solar (ciclo 24), conhecido como "tempestade do solstício de 2015". Esta tempestade geomagnética está associada a um período em que a Terra foi atingida por 4 CMEs. O painel direito da Figura 2 mostra que esta tempestade geomagnética atingiu a condição de $\mathrm{Kp}=8$ (grave). $\mathrm{O}$ índice $\mathrm{Kp}$ é baseado na média da componente horizontal do campo magnético da Terra realizada por 13 estações de magnetômetros que estão situadas ao longo do equador terrestre. O painel esquerdo da Figura 2 mostra uma queda na contagem do telescópio $\mathrm{New}$-Tupi vertical de forma bem relacionadas com dados do monitor de nêutrons situado no polo Sul e também com a sequência dos impactos provocados pelas CMEs. A queda na taxa de contagem do telescópio é esperada, conhecida como decréscimo Forbush [Augusto et al. 2012b] e está associada às tempestades geomagnéticas. Em geral, quanto mais intensa é a tempestade geomagnética, maior a queda na taxa de contagem de um detector de partículas ao nível do solo.
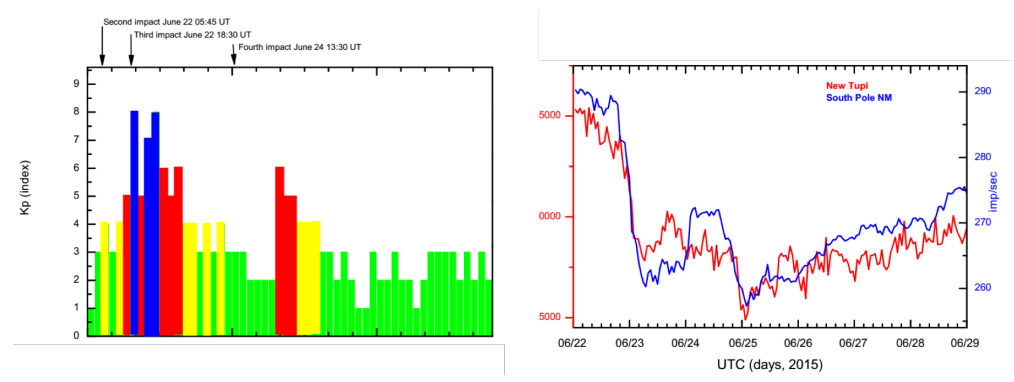

Figura 2. Painel esquerdo: Estimativa do índice planetário Kp (dados de 3 horas) por sete dias consecutivos, com início em 22 de junho de 2015 às 00:00 UT. Painel direito: Perfis temporais da taxa de contagem, para o mesmo período

\section{Abordagem Proposta: TupiDM}

Nessa seção apresentamos a abordagem proposta nesse artigo chamada TupiDM. 


\subsection{Cenário Atual}

Atualmente, os dados capturados pelos telescópios New-Tupi são armazenados em formato binário em um repositório nuvem. As medições são fragmentadas em arquivos diários, com extensões .DAT. Os arquivos se encontram organizados por ano, mês e dia, com a nomenclatura DB_Tupi _"Ano"_,Mes",'Dia". O conteúdo do arquivo é separado por tabulações, sendo a primeira coluna correspondente ao momento da medição representado no formato de tempo universal (número de segundos decorridos a partir de 01/01/1900). A segunda coluna corresponde às contagens para o telescópio vertical e a última coluna corresponde a soma das contagens dos telescópios inclinados (escaler). Atualmente, cabe aos físicos, localizar os arquivos desejados, realizar o download e calcular as agregações necessárias, de acordo com o período de tempo que se deseja analisar. Esse agrupamento hoje deve ser feito via script ou planilhas, o que não é escalável.

\subsection{Modelo de Dados do TupiDM}

A Figura 3 apresenta o Modelo do TupiDM. Foi utilizada a modelagem dimensional estrela [Kimball and Ross 2002], sendo a tabela fato representada pela tabela FAT_SINAIS, responsável por armazenar os fatos, que no contexto desse artigo são as contagens de sinais coincidentes detectados no detector vertical e no escaler, armazenados, respectivamente, nos atributos valorVertical e valorEscaler. Ainda na tabela FAT_SINAIS, os atributos idTempo e idTelescopio são chaves estrangeiras para as tabelas que representam as dimensões tempo e telescópio, respectivamente.
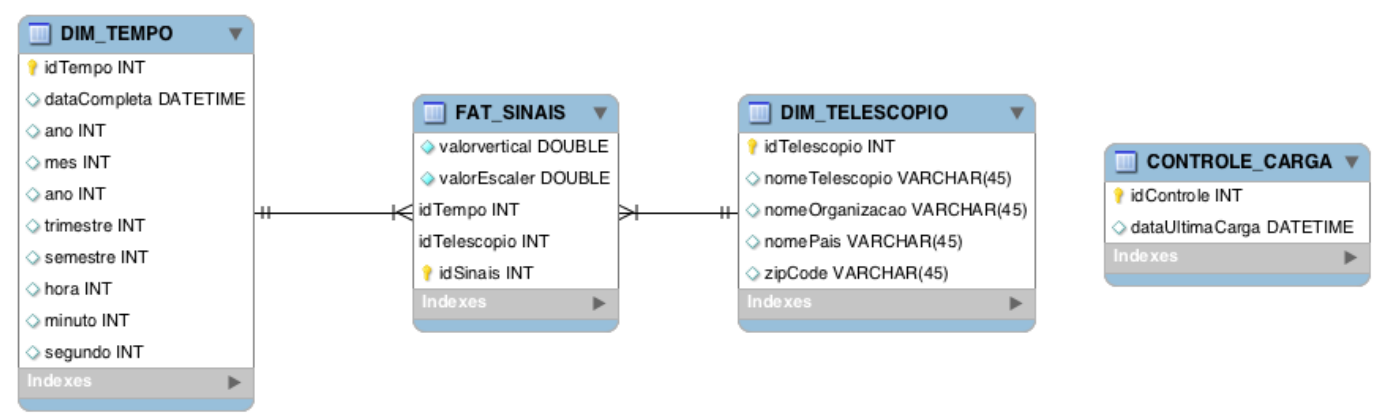

Figura 3. Esquema Estrela do TupiDM

A tabela DIM_TEMPO representa a dimensão tempo, sendo responsável por armazenar todas as possíveis granularidades de tempo para um determinado fato. $\mathrm{O}$ atributo dataCompleta representa a data completa (até milissegundos). Os atributos ano, mes, dia, trimestre, semestre, hora, minuto e segundo são as representações numéricas de partes da data completa. O atributo idTempo é a chave primária da tabela. A tabela DIM_TELESCOPIO representa a dimensão telescópio, sendo responsável por armazenar todas os possíveis grupos de telescópios para um fato. Ela é capaz de armazenar dados de outros telescópios de múons além dos $\mathrm{New}$-Tupi, de forma a permitir comparações entre medições de diferentes telescópios de múons. Porém, no momento contém apenas o New-Tupi. Ela possui os atributos idTelescopio, nomeTelescopio, nomeOrganizacao, nomePais e zipCode, que armazenam, respectivamente, chave primária da tabela, nome do grupo de telescópio, nome da organização ao qual o telescópio pertence, o país sede e a zona de informação postal.

\subsection{Arquitetura Proposta}

A Figura 4 apresenta a arquitetura proposta, desde o processo de captação dos dados pelos telescópios até a carga no DM proposto. A arquitetura proposta segue o tradicional ciclo 
de carga de um DW [Inmon 1992]. Os arquivos com as leituras vertical e escaler gerados pelos telescópios New-Tupi são armazenados no repositório remoto do Google Drive, que chamaremos somente de Drive a partir desse momento. Diariamente o componente ETL (Extract, Transform, Load), implementado por meio de um script Python, é executado. Esse script verifica se existem atualizações ou novos arquivos no Drive, e, caso haja, a mesma realiza o processo de carga no TupiDM. O TupiDM foi implementado no Sistema de Gerência de Banco de Dados PostgreSQL versão 9.6, instalado em um servidor com o sistema operacional Linux, distribuição Mint. O script python responsável pelo ETL foi agendado para ser diariamente executado com o cron do Linux.

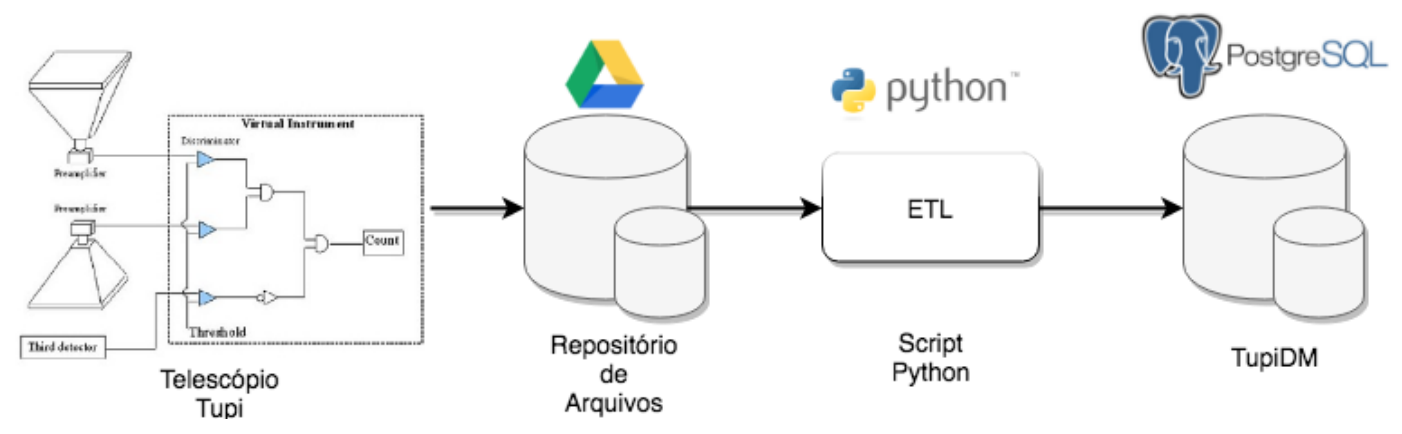

Figura 4. Arquitetura proposta para carga do TupiDM

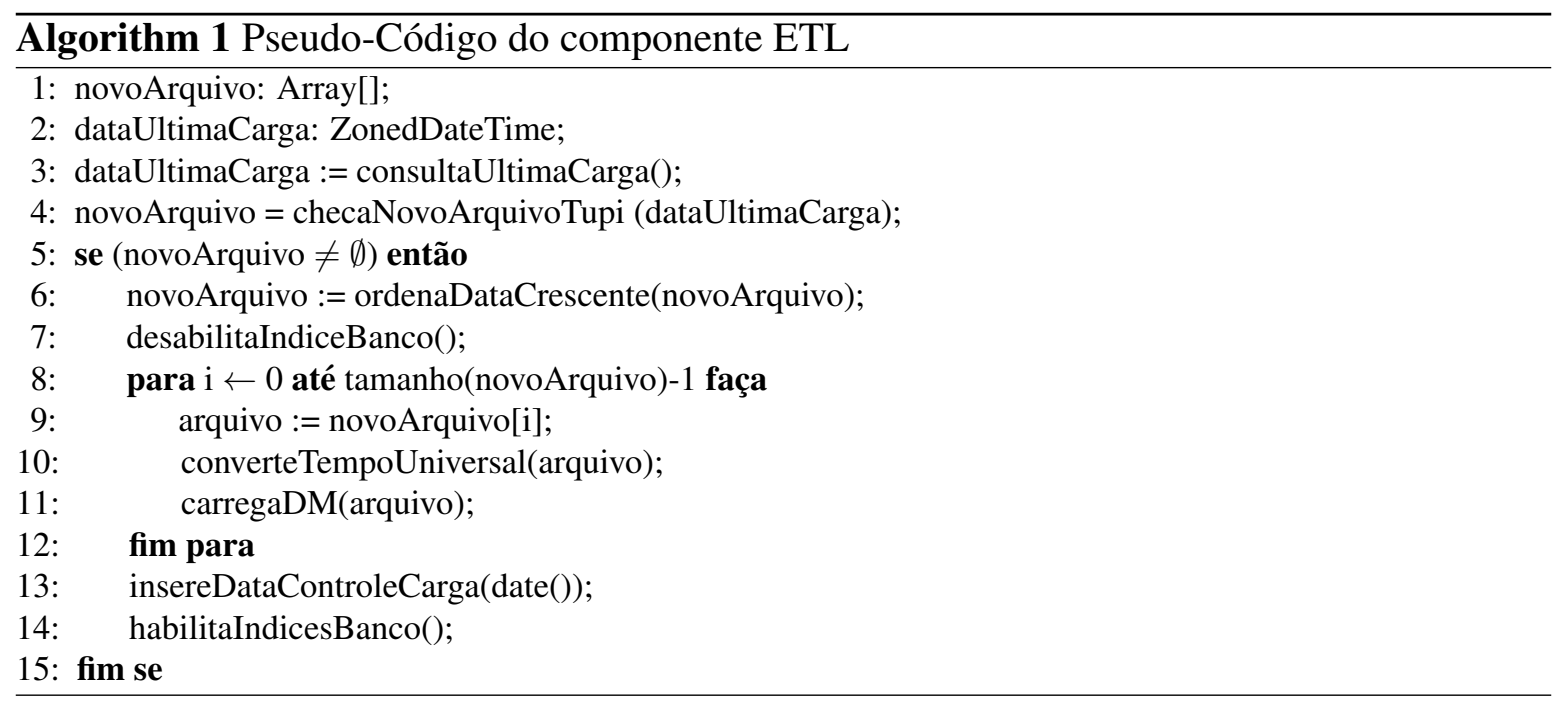

No Algoritmo 1 observamos os principais procedimentos realizados pelo componente ETL. Primeiramente, o componente consulta na tabela CONTROLE_CARGA do TupiDM quando foi feita a última carga de dados. Utilizando-se da data retornada pela consulta, o script busca no Drive por novos arquivos ou atualizações nos arquivos gerados pelo telescópio New-Tupi. Se houver resultados, os arquivos novos/atualizados são ordenados de forma crescente a partir de sua data de atualização, para que ao serem carregados na máquina, os arquivos mais atuais sobrescrevam os mais antigos na pasta, eliminando assim duplicatas. Os índices criados no TupiDM são desabilitados para agilizar o processo de carga. Para cada arquivo é verificado se já existem medições para aquele dia no TupiDM, e, caso haja, todos os registros correspondentes ao dia que está sendo inserido são excluídos, dessa forma garantimos que não há duplicidade de dados. Posteriormente, para cada linha contida nos arquivos a serem carregados é realizada a transformação do valor correspondente ao "tempo universal" para um formato de data, hora, minutos e segundos. Depois da transformação 
Tabela 1. Desempenho das consultas no TupiDM

\begin{tabular}{|c|c|c|}
\hline Consulta & S/ agregações & C/ agregações \\
\hline $\begin{array}{c}\text { Consultar o somatório dos valores das leituras verticais } \\
\text { e escaler durante o ano de } 2017\end{array}$ & $\begin{array}{l}297.467 \mathrm{~ms} \\
\text { (aprox. } 5 \mathrm{~min} \text { ) }\end{array}$ & $352 \mathrm{~ms}$ \\
\hline $\begin{array}{c}\text { Consultar o somatório dos valores das leituras verticais } \\
\text { e escalar no dia } 02 / 06 / 2016\end{array}$ & $\begin{array}{c}192.390 \mathrm{~ms} \\
\text { (aprox. 3,5 min) }\end{array}$ & $121 \mathrm{~ms}$ \\
\hline Consultar todas as leituras realizadas no dia $02 / 06 / 2016$ & $2.432 \mathrm{~ms}$ & $2.567 \mathrm{~ms}$ \\
\hline
\end{tabular}

a linha é inserida no TupiDM de acordo com a modelagem apresentada na Seção 3.2 e os agregados afetados têm seus valores atualizados. Ao término da carga, os índices são habilitados novamente e é inserida a data e hora, atuais na tabela CONTROLE_CARGA.

\section{Avaliação Experimental}

De forma a avaliar a abordagem proposta nesse artigo, realizamos uma avaliação experimental com uma amostra de 754 arquivos de leituras do telescópio New-Tupi. Esses arquivos correspondem às medições no período compreendido entre os anos 2014 e 2017. Após a execução do componente ETL, foram carregados apenas 750 arquivos no TupiDM, pois o algoritmo de carga descartou 4 arquivos duplicados. A tabela de fatos FAT_SINAIS possui um total de 33.311 .791 de registros e a tabela da dimensão tempo DIM_TEMPO, também possui um total de 33.311.791 de registros, uma vez que existe uma leitura do telescópio para cada unidade de tempo representada. O TupiDM sem índices criados ocupou um espaço em disco de aproximadamente 4 GB, e com índices criados, um espaço aproximado de $12 \mathrm{~GB}$.

A Tabela 1 exibe três consultas e seus tempos de execução associados (por limitações de espaço apresentamos apenas três consultas). Para cada consulta apresentamos o tempo de execução com agregações pré-calculadas e sem agregações pré-calculadas. Na primeira consulta foi explorada a agregação dos valores dos campos de leitura vertical e escaler durante todo o ano de 2017. A segunda consulta explora agregação dos valores de leitura vertical e escaler para um único dia. Ambas consultas se beneficiam de agregações pré-calculadas presentes no TupiDM e, por isso, apresentam grande variação no tempo de execução com o uso e sem o uso de agregações pré-calculadas. A terceira consulta lista todas as leituras em um dia. Como essa consulta não possui agregação, as agregações pré-calculadas não influenciam seu desempenho. É importante ressaltar que a amostra contém apenas 750 arquivos, e os tempos de execução tendem a aumentar quando todos os dados estiverem carregados no TupiDM. Este experimento reforça a necessidade de se utilizar agregações pré-calculadas.

\section{Trabalhos Relacionados}

Algumas soluções existentes já exploram a aplicação de bancos de dados para representar o fluxo de múons [Verducci 2007]. Entretanto, esses bancos de dados são comumente disponibilizados em arquivos .CSV para os físicos, o que limita a capacidade analítica dos mesmos. Nenhuma das abordagens existentes provê acesso a um banco de dados "consultável" e nem que possua capacidades analíticas de um DM. [Verducci 2007] propõe um banco de dados para o fluxo de múons do CERN (Organização Europeia para a Pesquisa Nuclear). [Verducci 2007] afirma que o uso de agregações pré-calculadas pode acelerar a pesquisa, principalmente para dados em larga-escala. Similarmente, o Cosmic Ray Muon Database ${ }^{2}$ também provê acesso a um dataset de leituras de múons em formato .CSV, o que também limita a capacidade analítica.

\footnotetext{
${ }^{2}$ http://cosray.shinshu-u.ac.jp/crest
} 


\section{Conclusões e Trabalhos Futuros}

Os telescópios New-Tupi realizam leituras do fluxo de múons que chega ao nosso planeta. A análise desse fluxo pode identificar erupções solares, que são eventos que podem causar suspensões de atividades eletromagnéticas. Atualmente, todas as leituras realizadas pelos telescópios são armazenadas em arquivos binários. Dado o grande volume de dados que são gerados, consultar e analisar tais dados em arquivos se tornou uma tarefa tediosa e propensa a erros.

Nesse artigo, propomos o uso de um Data Mart para os dados capturados pelos telescópios New-Tupi chamado TupiDM. O TupiDM segue uma modelagem dimensional do tipo estrela [Kimball and Ross 2002] que permite que dados sejam armazenados de forma pré-calculada no TupiDM, acelerando assim consultas que eram bastante lentas anteriormente. Foi realizada uma avaliação experimental do TupiDM utilizando-se um sub-conjunto dos dados gerados (33.311.791 registros de leituras) e constatou-se que em algumas consultas a redução no tempo da consulta chegou a ordens de grandeza.

Como sugestões para trabalhos futuros, pode-se apontar a elaboração de uma aplicação Web que visa oferecer visualizações sobre os dados do TupiDM, que apresente gráficos de series temporais dinâmicos, de acordo com os filtros de tempo oferecidos. Além disso, uma aplicação Web também permitirá aos pesquisadores visualizar e analisar esses dados de qualquer lugar. Outro trabalho futuro interessante seria o estudo de padrões nos dados do TupiDM, utilizando-se de técnicas de mineração de dados, o que pode gerar novas descobertas na área.

\section{Referências}

Augusto, C. R. A., Kopenkin, V., Navia, C. E., Tsui, K. H., Shigueoka, H., Fauth, A. C., Kemp, E., Manganote, E. J. T., de Oliveira, M. A. L., Miranda, P., Ticona, R., and Velarde, A. (2012a). Variations of the muon flux at sea level associated with interplanetary icmes and corotating interaction regions. The Astrophysical Journal, 759(2):143.

Augusto, C. R. A., Kopenkin, V., Navia, C. E., Tsui, K. H., and Sinzi, T. (2012b). Search for a simultaneous signal from small transient events in the pierre auger observatory and the tupi muon telescopes. Phys. Rev. D, 86:022001.

Augusto, C. R. A., Navia, C. E., de Oliveira, M. N., Nepomuceno, A. A., Kopenkin, V., and Sinzi, T. (2017). Muon excess at sea level during the progress of a geomagnetic storm and high-speed stream impact near the time of earth's heliospheric sheet crossing. Solar Physics, 292(8):107.

Golfarelli, M. and Rizzi, S. (2009). Data Warehouse Design: Modern Principles and Methodologies. McGraw-Hill, Inc., New York, NY, USA, 1 edition.

Inmon, W. H. (1992). Building the Data Warehouse. John Wiley \& Sons, Inc., New York, NY, USA.

Kimball, R. and Ross, M. (2002). The Data Warehouse Toolkit: The Complete Guide to Dimensional Modeling. John Wiley \& Sons, Inc., New York, NY, USA, 2nd edition.

Lattes, C. M. G., Occhialini, G. P. S., and Powell, C. F. (1947). Observations on the Tracks of Slow Mesons in Photographic Emulsions. 2. Nature, 160:486-492. [,103(1947)].

Verducci, M. (2007). Atlas conditions database and calibration streams. Nucl. Phys. B (Proc.Suppl.), 172:250.

Zavattini, E. (1975). Section 5 - muon capture. In Hughes, V. W. and Wu, C., editors, Muon Physics, pages $219-261$. Academic Press. 